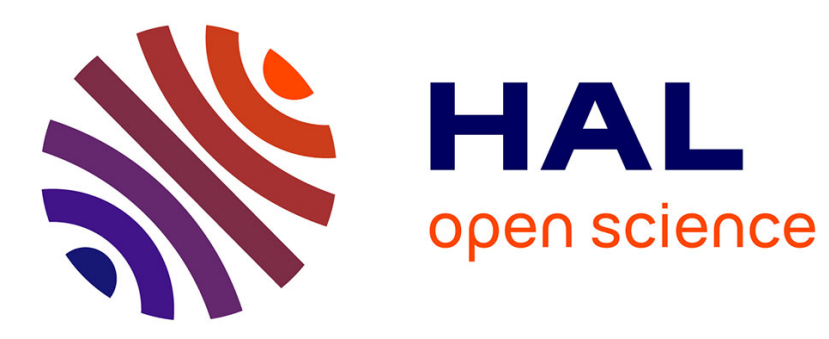

\title{
Interatomic Coulombic Decay Mediated by Ultrafast Superexchange Energy Transfer
}

Tsveta Miteva, Sévan Kazandjian, Přemysl Kolorenč, Petra Votavová, Nicolas Sisourat

\section{- To cite this version:}

Tsveta Miteva, Sévan Kazandjian, Přemysl Kolorenč, Petra Votavová, Nicolas Sisourat. Interatomic Coulombic Decay Mediated by Ultrafast Superexchange Energy Transfer. Physical Review Letters, 2017, 119 (8), pp.083403. 10.1103/PhysRevLett.119.083403 . hal-01667744

\section{HAL Id: hal-01667744 https: / hal.sorbonne-universite.fr/hal-01667744}

Submitted on 19 Dec 2017

HAL is a multi-disciplinary open access archive for the deposit and dissemination of scientific research documents, whether they are published or not. The documents may come from teaching and research institutions in France or abroad, or from public or private research centers.
L'archive ouverte pluridisciplinaire HAL, est destinée au dépôt et à la diffusion de documents scientifiques de niveau recherche, publiés ou non, émanant des établissements d'enseignement et de recherche français ou étrangers, des laboratoires publics ou privés. 


\title{
Interatomic Coulombic decay mediated by ultrafast superexchange energy transfer
}

\author{
Tsveta Miteva, ${ }^{1, *}$ Sévan Kazandjian, ${ }^{1}$ Přemysl Kolorenč,${ }^{2}$ Petra Votavová, ${ }^{2}$ and Nicolas Sisourat ${ }^{1}$ \\ ${ }^{1}$ Sorbonne Universités, UPMC Univ Paris 06, UMR 7614, \\ Laboratoire de Chimie Physique Matière et Rayonnement, \\ F-75005 Paris, France \\ ${ }^{2}$ Charles University, Faculty of Mathematics and Physics, \\ Institute of Theoretical Physics, V Holešovičkách 2, 18000 Prague, Czech Republic
}

(Dated: December 19, 2017)

\begin{abstract}
Inner-valence ionized states of atoms and molecules live shorter if these species are embedded in an environment due to the possibility for ultrafast de-excitation known as interatomic Coulombic decay (ICD). In this Letter we show that the lifetime of these ICD active states decreases further when a bridge atom is in proximity to the two interacting monomers. This novel mechanism, termed superexchange ICD, is an electronic correlation effect driven by the efficient energy transfer via virtual states of the bridge atom. The superexchange ICD is discussed in detail on the example of the NeHeNe trimer. We demonstrate that the decay width of the $\mathrm{Ne}^{+}\left(2 s^{-1}\right)^{2} \Sigma_{g}^{+}$resonance increases 6 times in the presence of the He atom at a distance of $4 \AA$ between the two Ne atoms. Using a simple model, we provide a qualitative explanation of the superexchange ICD and we derive analytical expressions for the dependence of the decay width on the distance between the neon atoms.
\end{abstract}

Excited atoms or molecules embedded in an environment can efficiently transfer their excess energy to neighboring species, whereupon the lifetime of the excited state decreases. Such processes of intermolecular energy transfer are ubiquitous in nature, for example, the first step of photosynthesis involves the transfer of energy from antenna complexes to reaction centres $[1,2]$ via the so-called Förster (or fluorescence) resonance energy transfer (FRET) [3]. FRET is accomplished through long-range Coulomb coupling between the interacting species and it involves transitions between bound states of the interacting chromophores. Owing to energy conservation this is only possible if nuclear motion is involved, which sets the time scale for this process to picoseconds or longer $[1,2]$.

If the excess energy of the excited species is larger than the ionization potential of the neighbor, another very efficient energy transfer mechanism, termed interatomic Coulombic decay (ICD), becomes operative. In this process, the energy is transferred to the neighbor which is then ionized [4]. Unlike FRET, ICD involves transitions to one or several continua of states and consequently, the requirement for energy conservation is fulfilled without the involvement of nuclear degrees of freedom [5]. This sets the time scale for ICD to femtoseconds [6-8].

The rate of ICD depends on the distance between the interacting species. If the coupling between the monomers is weak, which is the case of large distances, the process can be viewed as an exchange of a virtual photon between the interacting species $[9,10]$. In this so-called virtual photon exchange mechanism [11] the decay width displays a $1 / R^{6}$ dependence on the distance $R$ between the monomers. As the two monomers approach each other, orbital overlap effects come into play and,

\footnotetext{
* tsveta.miteva@upmc.fr
}

therefore, one can no longer view the ICD process simply as the exchange of a virtual photon [10].

A missing page in the study of ICD is how the energy transfer between the two monomers is influenced by the presence of ICD inactive neighbors, i.e. atoms or molecules whose ionization potential is greater than the excess energy of the excited species. In this Letter we show that the ICD process becomes faster when inert bridge atoms are added between the interacting monomers. As a showcase system we consider the deexcitation of the $\mathrm{Ne}^{+}\left(2 s^{-1}\right) \mathrm{Ne}^{2} \Sigma_{g}^{+}$resonance in the presence of a helium atom and compare the decay width to that of the well-studied isolated $\mathrm{Ne}$ dimer. As shown in Ref. [12], ICD between $\mathrm{Ne}$ and $\mathrm{He}$ is energetically closed for the interatomic distances considered here. We demonstrate that after inner-valence ionization of $\mathrm{Ne}$ in NeHeNe, the lifetime of the $\mathrm{Ne}^{+}\left(2 s^{-1}\right)^{2} \Sigma_{g}^{+}$resonance decreases from $832 \mathrm{fs}$ to $144 \mathrm{fs}$ at a distance of $4 \AA$ between the Ne atoms making ICD 6 times faster in the presence of the bridge atom. In FRET, energy transfer mediated by bridge molecules or bonds is known as superexchange mediated coupling [13]. We thus call the new ICD mechanism superexchange ICD. With the aid of a simple model, we show that this enhancement occurs due to coupling of the resonance state to intermediate virtual states of the bridge $\mathrm{Ne}^{+}\left(2 p^{-1}\right) \mathrm{He}^{-} \mathrm{Ne}^{+}\left(2 p^{-1}\right)$. The coupling depends on the energy difference between the resonance and the intermediate states, on the one hand, and on the orbital overlap between the virtual states of $\mathrm{He}$ and the bound states of $\mathrm{Ne}_{2}$, on the other hand.

The decay widths of $\mathrm{NeHeNe}$ and $\mathrm{Ne}_{2}$ were computed using the Fano-ADC method [14-16]. We employed the d-aug-cc-pVQZ basis set $[17,18]$ augmented with 15 continuumlike Gaussian functions (5s, 5p, 5d) of the Kaufmann-Baumeister-Jungen (KBJ) type [19] on all atoms. Additional sets of 5s, 5p and 5d KBJ functions were added on 4 ghost centers on the Ne-Ne inter- 


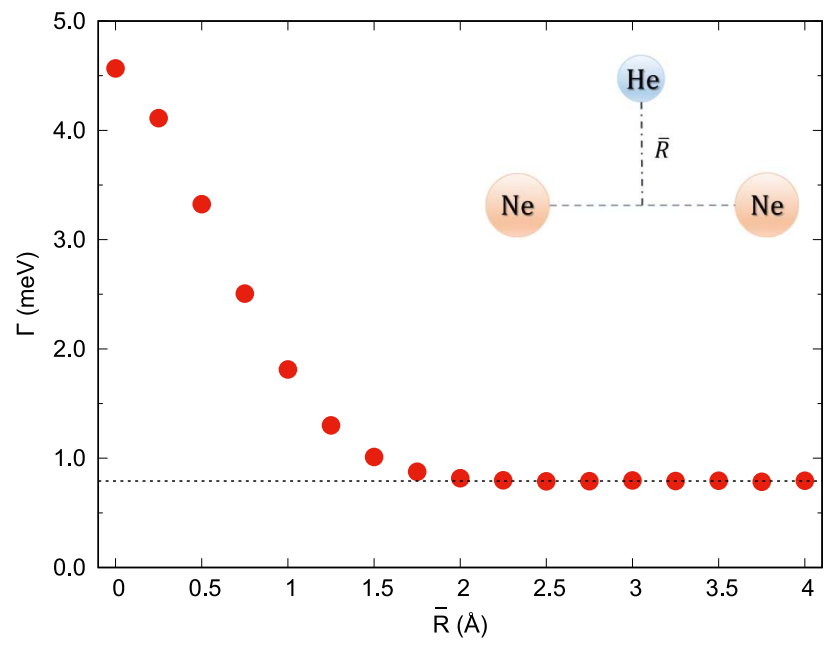

FIG. 1. Total decay width of the $\mathrm{Ne}^{+}\left(2 s^{-1}\right) \mathrm{HeNe}^{2} \Sigma_{g}^{+}$state as a function of the distance $\bar{R}$ between the He atom and the center of mass of neon dimer. The distance between the two neon atoms $R$ is fixed to $4 \AA$. The total decay width of the corresponding state of $\mathrm{Ne}_{2}$ is shown as black dotted line.

atomic axis at distance $\pm R / 4$ from each $\mathrm{Ne}$ atom, where $R$ is the distance between the two Ne atoms. The input Hartree-Fock data needed for the computation of the decay widths was generated with the MOLCAS quantum chemistry package [20]. In all calculations the occupancy of the $1 \mathrm{~s}$ orbital of He was kept fixed to 2 .

The ICD process after inner-valence ionization of isolated $\mathrm{Ne}_{2}$ has been extensively studied both theoretically and experimentally $[5,7,15,21,22]$. The lifetime of the $\mathrm{Ne}^{+}\left(2 s^{-1}\right) \mathrm{Ne}$ resonance was experimentally determined to be $150 \pm 50 \mathrm{fs}$ [7]. This result agrees very well with the mean lifetime (averaged over ${ }^{2} \Sigma_{g}^{+}$and ${ }^{2} \Sigma_{u}^{+}$states) of 158 fs computed at the equilibrium distance $(3.2 \AA)$ with the Fano-ADC method as described above.

Fig. 1 shows the total decay width of the $\mathrm{Ne}^{+}\left(2 s^{-1}\right)$ ${ }^{2} \Sigma_{g}^{+}$resonance in $\mathrm{NeHeNe}$ as a function of the distance $\bar{R}$ between He and the center of mass of the dimer. The distance between the two Ne atoms is kept constant, $R=$ $4 \AA$. As can be seen from Fig. 1, when the He atom approaches the center of mass of the dimer (see inset of Fig. 1), the total decay width of the $\mathrm{Ne}^{+}\left(2 s^{-1}\right) \mathrm{Ne}$ resonance increases. The effect becomes manifest as $\mathrm{He}$ is $1.75 \AA$ away from the center of mass of $\mathrm{Ne}_{2}$. When the He atom is aligned with the two neon atoms, the decay width is about 6 times higher compared to the decay width of the $\mathrm{Ne}^{+}\left(2 s^{-1}\right) \mathrm{Ne}^{2} \Sigma_{g}^{+}$state.

Next, we present the total decay width of the $\mathrm{Ne}^{+}\left(2 s^{-1}\right) \mathrm{HeNe}{ }^{2} \Sigma_{g}^{+}$resonance as a function of the distance between the Ne atoms keeping He at the center of mass of Ne dimer (see Fig. 2). It is compared with that of $\mathrm{Ne}_{2}$ where the He atom is replaced by a ghost atom with the same set of basis functions at the midbond position. As one can see, the two decay widths coincide at asymptotic distances, i.e. $R>7 \AA$. At shorter dis-

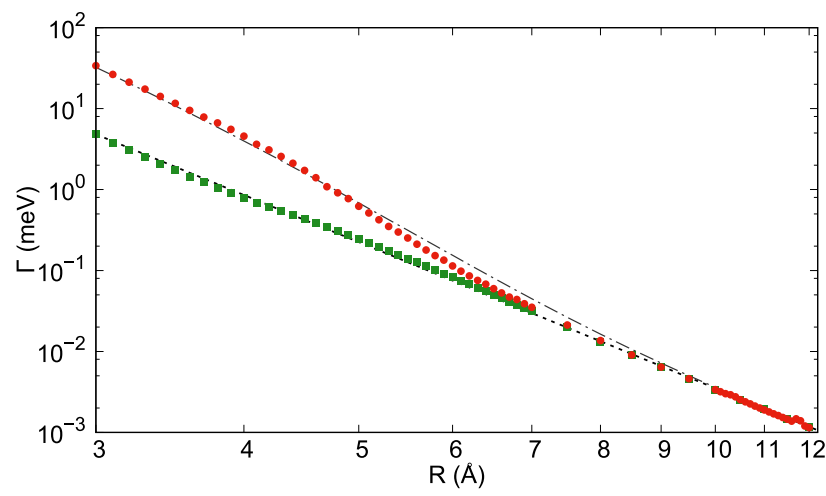

FIG. 2. Total decay widths of the ${ }^{2} \Sigma_{g}^{+}$state of $\mathrm{Ne}^{+}\left(2 s^{-1}\right) \mathrm{HeNe}$ (red) and $\mathrm{Ne}^{+}\left(2 s^{-1}\right) \mathrm{Ne}$ (green) as a function of the distance $R$ between the two Ne atoms, with He kept at the center of mass of the Ne dimer. The virtual photon approximation $[9,10]$ is shown as black dashed line scaled asymptotically to match the value of the theoretical decay width. The dashed-dotted line represents the analytical expression for the decay width in the case of superexchange ICD (see Eq. (7) in the text).

tances, the decay width of $\mathrm{NeHeNe}$ becomes increasingly higher than that of $\mathrm{Ne}_{2}$. Thus, the superexchange ICD mechanism becomes operative at distances below $7 \AA$.

The enhancement of the ICD width in the presence of a bridge atom can be understood from the energy level diagram in Fig. 3. The energies of all states shown on the diagram were computed as follows. First, the energies of the resonance and the final dicationic states were estimated using Koopmans' theorem. Second, we computed the energy of the $\mathrm{Ne}^{+}\left(2 p^{-1}\right) \mathrm{He}^{-} \mathrm{Ne}^{+}\left(2 p^{-1}\right)$ states as the energy of $\mathrm{He}^{-}$with two point charges at the position of the Ne atoms shifted by two times the $2 p$ ionization potential of Ne. The energies of $\mathrm{He}^{-}$were obtained using the Configuration Interaction Singles (CIS) method as implemented in the GAMESS-US computational package [23]. Finally, all states were shifted in energy such that the energy of the $\mathrm{Ne}^{+}\left(2 s^{-1}\right) \mathrm{HeNe}$ resonance matches the atomic value taken from NIST [24].

In its ground state the isolated He cannot bind an electron to form $\mathrm{He}^{-}[25,26]$. However, in the presence of two neighboring cations, the $\mathrm{He}^{-}\left(1 s^{2} n l\right)$ anion is stable, $\mathrm{Ne}^{+}\left(2 p^{-1}\right) \mathrm{He}^{-} \mathrm{Ne}^{+}\left(2 p^{-1}\right)$ states below $45.7 \mathrm{eV}$ in Fig. 3. As one can see from the figure, there are two pathways from the initial inner valence ionized $\mathrm{Ne}^{+}\left(2 s^{-1}\right) \mathrm{HeNe}$ state to the final doubly ionized states $\mathrm{Ne}^{+}\left(2 p^{-1}\right) \mathrm{HeNe}^{+}\left(2 p^{-1}\right)$ - direct and superexchange ICD. In the latter process, the transition from the initial to the final states occurs through coupling to intermediate ionic configurations of the type $\mathrm{Ne}^{+}\left(2 p^{-1}\right) \mathrm{He}^{-} \mathrm{Ne}^{+}\left(2 p^{-1}\right)$, which include virtual states of the bridge. The denotation "direct ICD" is used here to distinguish ICD in the isolated neon dimer from the superexchange ICD. It should not be confused with the direct integral in the approximate expression for the ICD width [27] whose expansion in powers of the distance $R$ 


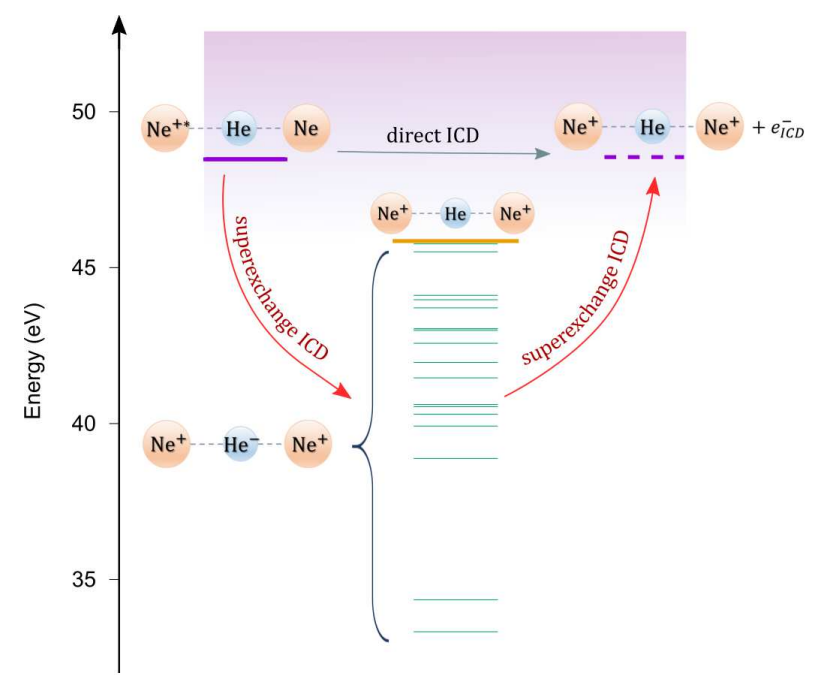

FIG. 3. Energy level diagram representing the direct and superexchange ICD processes. The initial and final ICD states (purple) are shown to the left and right of the diagram, respectively. The purple shaded area indicates that the states are in the continuum. The superexchange ICD process occurs via coupling of the initial states to intermediate ionic configurations, $\mathrm{Ne}^{+}\left(2 p^{-1}\right) \mathrm{He}^{-} \mathrm{Ne}^{+}\left(2 p^{-1}\right)$ (green). The ionization threshold of $\mathrm{Ne}^{+}\left(2 p^{-1}\right) \mathrm{He}^{-} \mathrm{Ne}^{+}\left(2 p^{-1}\right)$ is also shown (orange). All states have been computed for distance $4 \AA$ between the $\mathrm{Ne}$ atoms and the states have been shifted to the energy of the atomic $\mathrm{Ne}^{+}\left(2 s^{-1}\right)$ state taken from NIST [24].

between the interacting monomers gives the asymptotic $1 / R^{6}$ dependence of the decay width.

In order to provide a qualitative explanation of the superexchange ICD and the dependence of the decay width on the distance $R$ between the interacting monomers, in what follows, we derive an approximate expression for the ICD width as a function of $R$. Within the Fano formalism, the total decay width is a sum over the partial decay widths corresponding to different $\left(N_{c}\right)$ decay channels $[28,29]$

$$
\Gamma=2 \pi \sum_{\beta}^{N_{c}}\left|\left\langle\Psi_{R}\left|\hat{H}-E_{R}\right| \Psi_{P}^{\beta}\right\rangle\right|^{2}=2 \pi \sum_{\beta}^{N_{c}}\left|H_{R P}^{\beta}\right|^{2}
$$

where $\Psi_{R}$ is the wave function of the decaying state, $\Psi_{P}^{\beta}$ are the final state wave functions, $E_{R}$ is the energy of the resonance, and the final states fulfill the resonance condition $E_{P}^{\beta}=E_{R}$. In order to evaluate the coupling matrix elements in Eq. (1) we use a configuration interaction approach developed by Scholes and Harcourt for the description of superexchange-mediated coupling in FRET $[13,30]$. We start by defining the initial $\psi_{i}$, final $\psi_{f}^{\beta}$ and intermediate states $\psi^{J}$ shown in Fig. 3 as the following configuration state functions

$$
\begin{aligned}
\psi_{i} & =\hat{A}\left|\Phi_{\mathrm{Ne}_{2}^{+} \Sigma_{g}^{+}}\right\rangle\left|\Phi_{\mathrm{He}}\right\rangle \\
\psi_{f}^{\beta} & =\hat{A}\left|\Phi_{\mathrm{Ne}_{2}^{++}}^{\beta}\right\rangle\left|\Phi_{\mathrm{He}}\right\rangle|k\rangle \\
\psi^{J} & =\hat{A}\left|\Phi_{\mathrm{Ne}_{2}^{++}}^{\gamma}\right\rangle\left|\Phi_{\mathrm{He}^{-}}^{m}\right\rangle
\end{aligned}
$$

Here the Greek letters $\beta, \gamma$ are used to label the different decay channels, i.e. the final dicationic states $\mathrm{Ne}^{+}\left(2 p^{-1}\right) \mathrm{HeNe}^{+}\left(2 p^{-1}\right)$; the superscript $m$ denotes the ionic states of $\mathrm{He}^{-}, J$ stands for the combination of indices $\gamma$ and $m ;|k\rangle$ describes the outgoing electron, and $\hat{A}$ is the antisymmetrization operator. Next, we construct the reactant $\Psi_{R}$ and product $\Psi_{P}^{\beta}$ wave functions needed to evaluate expression Eq. (1) as linear combinations of the configuration state functions (see Eq. (2))

$$
\begin{aligned}
& \Psi_{R}=\psi_{i} \\
& \Psi_{P}^{\beta}=\psi_{f}^{\beta}+\sum_{J} \frac{H_{J f}^{\beta}}{E_{f}^{\beta}-E_{J}} \psi^{J}
\end{aligned}
$$

Here assuming a weak coupling between the donor/acceptor and bridge configurations, the coefficients in front of the intermediate states $\left\{\psi^{J}\right\}$ are determined from first-order perturbation theory. Neglecting the interference between the intermediate channels $\psi^{J}$ and taking into account the resonance condition $\left(E_{i}=E_{f}^{\beta}=E_{r e s}\right)$, one obtains for the coupling matrix element $H_{R P}^{\beta}$

$$
H_{R P}^{\beta} \approx H_{i f}^{\beta}+\sum_{J} \frac{H_{i J} H_{J f}^{\beta}}{E_{r e s}-E_{J}}
$$

The first term on the r.h.s. corresponds to the direct ICD channel. The second term results from the coupling of the initial configuration $\psi_{i}$ with the intermediate ionic configurations $\psi^{J}$. The matrix element $H_{J f}^{\beta}$ can be regarded as the coupling between $\mathrm{He}^{-}\left(1 s^{2} n l\right)$ and $\mathrm{He}+e^{-}$ in the presence of two point charges.

By examining Eq. (5) we distinguish two extreme cases. In the first case, the direct ICD channel is predominant over the superexchange ICD. Then one can neglect the second term in Eq. (5) and the total decay width of $\mathrm{Ne}^{+}\left(2 s^{-1}\right) \mathrm{HeNe}$ becomes equal to that of the direct ICD channel, i.e. the decay width of $\mathrm{Ne}^{+}\left(2 s^{-1}\right) \mathrm{Ne}$ in the absence of He. As can be seen in Fig. 2, this is the case of $R>7 \AA$. At large distances between the two monomers, the virtual photon approximation to the ICD width (dashed black line in Fig. 2) is valid and thus, both decay widths exhibit the expected $1 / R^{6}$ behavior $[5,9]$.

In the second case, the superexchange ICD channel is predominant and the first term in Eq. (5) can be neglected. To quantify the $R$-dependence of the decay width in this case, we evaluate the matrix elements in the second term on the r.h.s. of Eq. (5) by considering a simple model where the wave functions $\psi_{i}, \psi_{f}^{\beta}$ and $\psi^{J}$ are 
represented as spin-adapted linear combinations of Slater determinants [30, 31]. The second term in Eq. (5) then becomes a sum of two-electron integrals. Expanding the two-electron integrals in a power series in $R$ as explained in detail in Ref. [27], we obtain the following expression for the coupling matrix element $H_{R P}^{\beta}$

$$
H_{R P}^{\beta}(R) \approx \sum_{J} B_{J f}^{\beta} \frac{1}{E_{r e s}-E_{J}} \frac{\left\langle\chi_{N e}^{J} \mid \chi_{H e}^{J}\right\rangle}{R^{2}}
$$

where $R$ is the distance between the two Ne atoms, and $\left\langle\chi_{N e}^{J} \mid \chi_{H e}^{J}\right\rangle$ is the overlap integral between occupied orbitals of $\mathrm{Ne}_{2}$ and unoccupied orbitals of He. For the range of distances considered here, the number of bridge states does not vary substantially. The coefficient $B_{J f}^{\beta}$ is a product of several terms: the coupling matrix element $H_{J f}^{\beta}$, a transition dipole moment term resulting from the power series expansion of the two-electron integral [27], and a factor resulting from the spin multiplicity of the final dicationic $\mathrm{Ne}^{+}\left(2 p^{-1}\right) \mathrm{HeNe}^{+}\left(2 p^{-1}\right)$ states. The overlap terms decrease exponentially with the interatomic distance between $\mathrm{Ne}$ and $\mathrm{He}$ while $B_{J f}^{\beta}$ contains terms that are of intraatomic nature and thus has a much weaker $R$-dependence than the overlaps. Therefore, we assume that $B_{J f}^{\beta}$ remains constant for the range of interatomic distances for which the superexchange mechanism is operative. As Eq. (6) shows, the superexchange mechanism kicks in as the overlap between the virtual states of the bridge and the bound states of $\mathrm{Ne}_{2}$ becomes non-negligible, and is suppressed at large distances between $\mathrm{Ne}$ and He. Owing to the $1 / R^{4}$ dependence of the superexchange decay width and since there are several intermediate states through which the superexchange mechanism can occur, it is expected to outpace the direct ICD in the typical range of distances of rare gas clusters.

It should be noted that excited configurations of the type $\mathrm{Ne}^{+}\left(2 p^{-1}\right) \mathrm{He}^{*} \mathrm{Ne}$ are excluded from the ansatz for the reactant and product wave functions (Eq. (3) and (4)) as well as in the Fano-ADC calculations. Using the CI model presented above, we found that their contribution to the ICD rate behaves like $1 / R^{12}$ and is thus expected to be negligible for most geometries. However, the coupling to the resonance is inversely proportional to the energy difference between the excited configurations and the resonance. For certain geometries their energy can be nearly degenerate with that of the resonance. As a result, the large energy factor can counterbalance the unfavorable $1 / R^{12}$ factor and therefore, this energy transfer pathway can become significant.

Neglecting the interference between the direct and superexchange ICD channels and approximating the sum of weighted overlaps in Eq. (6) as a single exponential, the expression for the total decay width as a function of the distance becomes

$$
\Gamma(R)=\sum_{\beta} \Gamma_{\beta} \approx \frac{A}{R^{6}}+B \frac{e^{-C R}}{R^{4}}
$$

The approximate expression for $\Gamma(R)$ is plotted in Fig. 2, where $A, B$ and $C$ are fitted to the theoretical decay width. As can be seen, the agreement between the computed total decay width of the $\mathrm{Ne}^{+}\left(2 s^{-1}\right) \mathrm{HeNe}^{2} \Sigma_{g}^{+}$ resonance and Eq. (7) is very good for the two extreme cases of large $(R>8 \AA)$ and small $(R<5 \AA)$ interatomic distances, where the superexchange or the direct ICD channel are predominant. A small deviation of the analytical expression from the theoretical decay width in the intermediate region between 5 and $8 \AA$ can be explained with the neglect of interference effects between the direct and superexchange channels in our analytical derivation.

In conclusion, we show that in the presence of environment which is ICD inactive, the ICD process becomes more efficient. The decrease of the ICD lifetime is a result of configuration interaction with intermediate states of the ICD inactive neighbors. This superexchange pathway occurs under two conditions. First, the intermediate states should lie in energetic proximity to the resonance. Second, the ICD active species and the bridge atom have to be close enough such that there is sufficient orbital overlap.

The superexchange ICD mechanism may be observed experimentally by comparing time-resolved experiments, such as in Refs. [7, 32], on Ne clusters doped with an increasing number of He atoms, on the one hand, and on homogeneous Ne clusters, on the other hand. Since these experiments rely on the detection of ions, it might be preferable to use small clusters in order to avoid charge transfer processes following ICD.

Finally, we would like to briefly discuss the significance of the superexchange ICD mechanism. First, adding ICD inactive atoms provides a way to control the relaxation processes following the deposition of energy into the system. For example, by enhancing the efficiency of the ICD process, one can suppress other processes, such as those involving nuclear rearrangements [33, 34]. Second, by adding an ICD inactive neighbor, one can transfer the excitation energy over larger distances and thus ionize more distant neighbors.

We thank Prof. L. S. Cederbaum for the fruitful discussions. This project has received funding from the Research Executive Agency (REA) under the European Union's Horizon 2020 research and innovation programme Grant agreement No. 705515. P. K. and P. V. acknowledge financial support by the Czech Science Foundation (Project GAČR No. 17-10866S).
[1] T. Renger, V. May, and O. Kühn, Phys. Rep. 343, 137 (2001).
[2] G. D. Scholes, G. R. Fleming, A. Olaya-Castro, and 
R. van Grondelle, Nat. Chem. 3, 763 (2011).

[3] T. Förster, Ann. Phys. 437, 55 (1948).

[4] L. S. Cederbaum, J. Zobeley, and F. Tarantelli, Phys. Rev. Lett. 79, 4778 (1997).

[5] R. Santra, J. Zobeley, L. S. Cederbaum, and N. Moiseyev, Phys. Rev. Lett. 85, 4490 (2000).

[6] W. Pokapanich, N. V. Kryzhevoi, N. Ottosson, S. Svensson, L. S. Cederbaum, G. Öhrwall, and O. Björneholm, J. Am. Chem. Soc. 133, 13430 (2011).

[7] K. Schnorr, A. Senftleben, M. Kurka, A. Rudenko, L. Foucar, G. Schmid, A. Broska, T. Pfeifer, K. Meyer, D. Anielski, R. Boll, D. Rolles, M. Kübel, M. F. Kling, Y. H. Jiang, S. Mondal, T. Tachibana, K. Ueda, T. Marchenko, M. Simon, G. Brenner, R. Treusch, S. Scheit, V. Averbukh, J. Ullrich, C. D. Schröter, and R. Moshammer, Phys. Rev. Lett. 111, 093402 (2013).

[8] F. Trinter, J. B. Williams, M. Weller, M. Waitz, M. Pitzer, J. Voigtsberger, C. Schober, G. Kastirke, C. Müller, C. Goihl, P. Burzynski, F. Wiegandt, R. Wallauer, A. Kalinin, L. P. H. Schmidt, M. S. Schöffler, Y.-C. Chiang, K. Gokhberg, T. Jahnke, and R. Dörner, Phys. Rev. Lett. 111, 233004 (2013).

[9] R. Santra, J. Zobeley, and L. S. Cederbaum, Phys. Rev. B 64, 245104 (2001).

[10] V. Averbukh, I. B. Müller, and L. S. Cederbaum, Phys. Rev. Lett. 93, 263002 (2004).

[11] J. Matthew and Y. Komninos, Surf. Sci. 53, 716 (1975).

[12] N. Sisourat, H. Sann, N. V. Kryzhevoi, P. Kolorenč, T. Havermeier, F. Sturm, T. Jahnke, H.-K. Kim, R. Dörner, and L. S. Cederbaum, Phys. Rev. Lett. 105, 173401 (2010).

[13] G. D. Scholes, Ann. Rev. Phys. Chem. 54, 57 (2003).

[14] V. Averbukh and L. S. Cederbaum, J. Chem. Phys. 123, 204107 (2005).

[15] V. Averbukh and L. S. Cederbaum, J. Chem. Phys. 125, 094107 (2006).

[16] P. Kolorenč, V. Averbukh, K. Gokhberg, and L. Cederbaum, J. Chem. Phys. 129, 244102 (2008).

[17] T. H. Dunning, J. Chem. Phys. 90, 1007 (1989).

[18] D. Woon and J. T. H. Dunning, J. Chem. Phys. 100,
2975 (1994).

[19] K. Kaufmann, W. Baumeister, and M. Jungen, J. Phys. B At. Mol. Opt. Phys. 22, 2223 (1989).

[20] F. Aquilante, L. De Vico, N. Ferré, G. Ghigo, P. Malmqvist, P. Neogrády, T. B. Pedersen, M. Pitoňák, M. Reiher, B. O. Roos, L. Serrano-Andrés, M. Urban, V. Veryazov, and R. Lindh, J. Comp. Chem. 31, 224 (2010).

[21] S. Scheit, V. Averbukh, H.-D. Meyer, N. Moiseyev, R. Santra, T. Sommerfeld, J. Zobeley, and L. S. Cederbaum, J. Chem. Phys. 121, 8393 (2004).

[22] N. Vaval and L. S. Cederbaum, J. Chem. Phys. 126, 164110 (2007).

[23] M. W. Schmidt, K. K. Baldridge, J. A. Boatz, S. T. Elbert, M. S. Gordon, J. H. Jensen, S. Koseki, N. Matsunaga, K. A. Nguyen, S. Su, T. L. Windus, M. Dupuis, and J. A. Montgomery, J. Comp. Chem. 14, 1347 (1993).

[24] A. Kramida, Y. Ralchenko, J. Reader, and N. T. (2016), NIST Atomic Spectra Database (version 5.4), [Online]. Available: http://physics.nist.gov/asd. National Institute of Standards and Technology, Gaithersburg, MD. (2017).

[25] T.-Y. Wu, Phil. Mag. 22 (1936).

[26] O. Tüxen, Z. Phys. 103, 463 (1936).

[27] R. Santra and L. S. Cederbaum, Phys. Rev. 368, 1 (2002).

[28] U. Fano, Phys. Rev. 124, 1866 (1961).

[29] G. Howat, T. Åberg, and O. Goscinski, J. Phys. B At. Mol. Opt. Phys. 11, 1575 (1978).

[30] R. D. Harcourt, G. D. Scholes, and K. P. Ghiggino, J. Chem. Phys. 101, 10521 (1994).

[31] G. D. Scholes and R. D. Harcourt, J. Chem. Phys. 104, 5054 (1996).

[32] U. Frühling, F. Trinter, F. Karimi, J. Williams, and T. Jahnke, J. Electron Spectrosc. Relat. Phenom. 204, 237 (2015).

[33] S. Kopelke, Y.-C. Chiang, K. Gokhberg, and L. S. Cederbaum, J. Chem. Phys. 137, 034302 (2012).

[34] G. Jabbari, K. Sadri, L. S. Cederbaum, and K. Gokhberg, J. Chem. Phys. 144, 164307 (2016). 